\title{
Supervisão e controlo de um sistema de canais de rega. Parte I - Definição e apresentação geral
}

\author{
Supervision and control of an irrigation canal system. \\ Part I - Definition and general presentation
}

\author{
Manuel Rijo ', Bruno Inácio 2, João Campos ${ }^{3}$ \\ ${ }^{1}$ Prof. Assoc. c/ Agregação U. Évora, membro da APRH no732, Escola de Ciência e Tecnologia, Universidade de Évora, \\ Instituto de Ciências Agrárias e Ambientais Mediterrânicas, Apartado 94, 7002-554 Évora, Portugal; e-mail: rijo@uevora.pt \\ ${ }^{2}$ Eng. ${ }^{\circ}$ Eletrotécnico, ACTEMIUM, Cegelec - Instalações e Sistemas de Automação Lda. \\ ${ }^{3}$ Eng ${ }^{\circ}$. Agrónomo, DGADR-Direção Geral de Agricultura e Desenvolvimento Rural
}

RESUMO: O artigo faz a apresentação geral do sistema de supervisão e controlo (SCADA) desenvolvido para as redes primária e secundária de canais do Aproveitamento Hidroagrícola de Idanha-a-Nova, já instalado e calibrado.

O SCADA permite melhorar, de modo significativo, a eficiência da gestão operacional dos canais de rega controlados por montante, tipo de canais do Aproveitamento, ao fazer o controlo de caudais nas admissões principais, a supervisão dos caudais perdidos nas descargas principais e das alturas de água nas secções de maior interesse para o controlo e a segurança. Ao atuar em tempo real e à distância, ganha-se tempo, melhora-se a segurança do funcionamento dos canais, aumenta-se a qualidade das distribuições e reduzem-se as perdas operacionais de água.

Para além da apresentação geral do SCADA, incluindo o centro de controlo, as comunicações e estações de campo, são também apresentados alguns dos sinópticos desenvolvidos e os controladores digitais instalados: direto para comporta, de posição de comporta e controlador de caudal para comporta.

Palavras-chave: Canais de rega; controlo por montante; comportas AMIL; tecnologia SCADA; modernização de canais.

ABSTRACT: The paper presents the developed supervisory control and data acquisition system (SCADA system) for the main and secondary canal network of the Idanha-a-Nova Irrigation District, already installed and tuned.

SCADA improves, significantly, the operational management efficiency of the irrigation canals upstream controlled, the type of canals that is installed in the Irrigation District, insuring the inflow control at the head of the main canals, the outflow supervision for the drainage system and the supervision of the water depths at the most important canal sections for control purposes and canals security. Acting in remote mode and real time, it saves time, improves the safety of the canals operation, increases the water delivery quality and reduces the operational water losses.

Besides the general SCADA presentation, including the control centre, communications and field stations, that are also presented a few of the developed synoptics and the installed digital controllers: gate direct controller, gate position controller and gate flow controller.

Keywords: Irrigation canals; upstream control; AMIL gates; SCADA technology; canal modernization.

Otexto deste artigo foi submetido para revisão e possível publicação em novembro de 2015, tendo sido aceite pela Comissão de Editores Científicos Associados em janeiro de 2016. Este artigo é parte integrante da Revista Recursos Hídricos, Vol. 37, № 1, 59-72, março de 2016.

๑ APRH, ISSN 0870-1741 |DOI 10.5894/rh37n1-cti2

Recursos Hídricos $\mid 59$ 


\section{INTRODUÇÃO}

Os grandes sistemas de adução de água bruta são habitualmente em canal (Rijo, 2010). Razões técnicas e económicas determinam esta opção habitual. É o caso da grande maioria dos Aproveitamentos Hidroagrícolas do País, com cerca de 200000ha de área infraestruturada (SIR, s/d), sobretudo nas redes primárias e, com menor expressão, nas redes secundárias. O mesmo acontece com praticamente toda a rede primária do Empreendimento de Fins Múltiplos do Alqueva, com cerca de $400 \mathrm{~km}$ de extensão e que serve cerca de 120000 ha (EDIA, s/d).

Estes canais nacionais estão equipados com o controlo por montante, o controlo que equipa mais de $90 \%$ dos canais de rega a nível mundial (Rijo, 2010). Tal acontece porque o dimensionamento dos canais pode ser otimizado (secção transversal constante ao longo do percurso e com uma altura que, para além da folga usual, praticamente corresponde à altura uniforme para o caudal de projeto, $Q=Q \max$, Figura 1b) e porque os equipamentos de controlo podem ser simples e económicos (Rijo, 2010).

Nas aplicações nacionais do controlo local por montante nos Aproveitamento Hidroagrícolas, o controlador mais usado é a comporta AMIL/ AMP (Figura 1a), uma comporta de segmento automática que, quando bem calibrada, impõe uma altura de água imediatamente a montante correspondente à cota de assentamento do seu eixo de rotação, independentemente do caudal em circulação e com uma precisão de cerca de 3-4\% da altura do respetivo tabuleiro, o chamado "décrément" (ALSTHOM).

A variável controlada no controlo local por montante é a altura de água a jusante de cada trecho de canal ou imediatamente a montante de cada controlador (Figura 1b). Quando há variação de caudal em circulação, a comporta ajusta a sua abertura para repor a variável controlada no seu valor de referência. O valor de referência é, por norma, a altura de água em regime uniforme para o caudal de dimensionamento do trecho $(Q=Q \max$, na

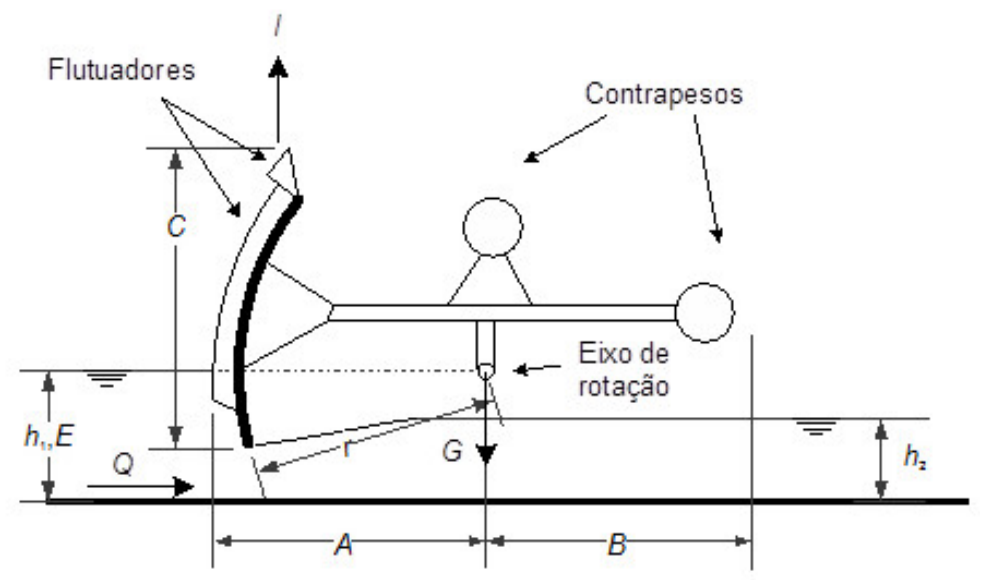

a) Comporta AMIL

b)

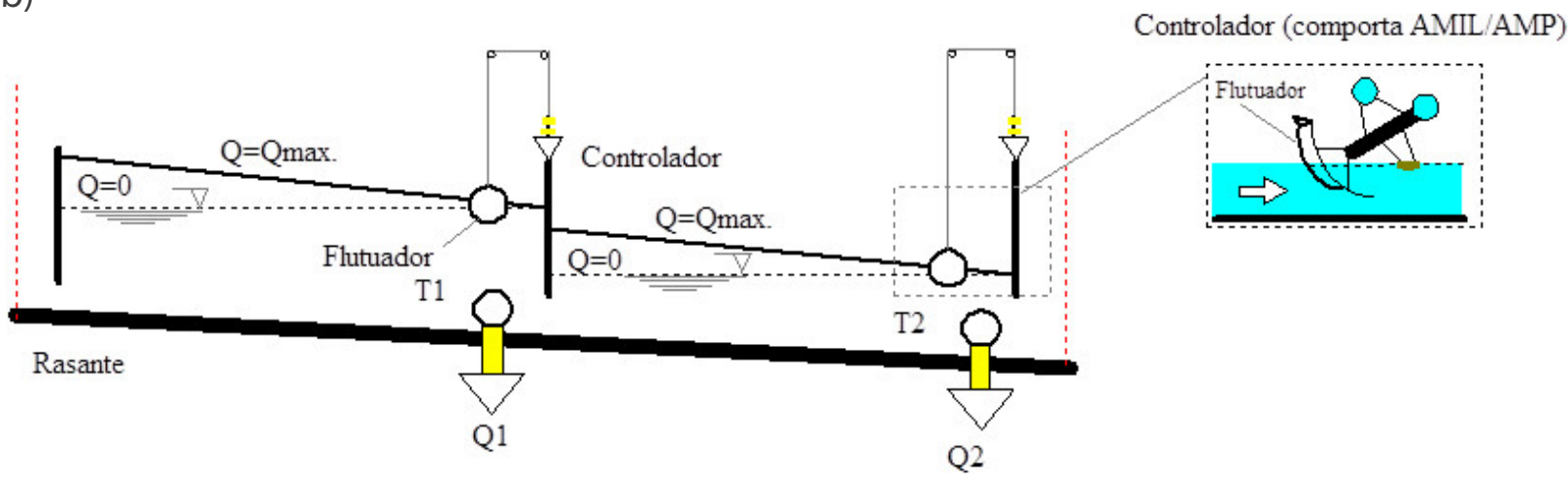

Figura 1. Controlo local por montante: a) comporta AMIL; b) Representação esquemática de dois trechos de canal com comportas AMIL (Rijo, 2008 a). 
Figura 1b), com um acréscimo para fazer face à perda de carga exigida pela comporta (sempre muito pequena, poucos $\mathrm{cms}$ ).

O controlo local por montante visa apenas o controlo de alturas de água no interior dos canais. Não permite o controlo de caudais. Por isso, exige sempre a ação complementar de um controlo, sempre manual, de caudais em todas as admissões aos canais.

Atendendo à dinâmica hidráulica de cada trecho de canal, este tipo de controlo adequase bem às variações de caudal a montante (admissão ao trecho) e responde de forma bastante ineficiente (com atraso e perdas de água consideráveis) às variações de caudal a jusante (seção de jusante do trecho ou nas tomadas de água).

Se a tomada de água T2 (Figura 1b) fechar, o seu caudal passa a ficar no trecho de canal, fazendo subir a cota da superfície livre, quando esta deveria descer (um menor caudal em circulação exige um menor volume de água armazenado no trecho, Figura 1b). A comporta de jusante vai ter de abrir para deixar passar o caudal que deixou de ser derivado na tomada T2 e, durante o regime transitório correspondente no trecho, também para deixar passar o acréscimo de caudal exigido pela diminuição do volume de água no trecho. A resposta do trecho de canal é lenta e dá origem a perdas de água importantes para jusante.

Por outro lado, se, instantaneamente, se abrisse a tomada de água T2, esta seria alimentada à custa da água armazenada no trecho de canal e este, se não for alimentado por montante com recurso a manobras na admissão em valor suficiente e realizadas com a antecipação necessária, vai esvaziar. Por isso, este tipo de controlo responde muito mal quando associado a métodos flexíveis de distribuição de água, em que as manobras nas diferentes tomadas de água são permitidas com maior ou menor liberdade.

Qualquer variação de caudal a montante do trecho de canal é muito mais facilmente acomodada, Se, por exemplo, houver instantaneamente um acréscimo de caudal na comporta de montante do trecho de canal, neste a cota da superfície livre começa a subir e, quando for igual à exigida pelo novo caudal em regime permanente, o trecho estabiliza e todo o caudal passa para jusante. Por outro lado, quando houver um decréscimo de caudal a montante, o trecho de canal vai ter de esvaziar parcialmente até atingir a superfície livre correspondente ao novo caudal em regime permanente em circulação e, neste caso, o esvaziamento corresponde a uma perda de água do trecho.

Idealmente, se não houver variações de caudal nem a montante nem a jusante, não haverá perdas de água no trecho de canal. $\mathrm{O}$ trecho de canal só é eficiente no uso da água nessa situação.

As variações (manuais) de caudal nas admissões respondem de modo muito ineficiente às variações de caudal nas tomadas de água, mesmo quando estas são conhecidas com antecipação suficiente. É impossível definir um hidrograma de caudais à cabeça de um canal que permita a satisfação da distribuição de água em todas as tomadas de água de forma otimizada. Quando as variações de caudal nas tomadas são imprevisíveis e até de sinais contrários, a ineficiência do sistema aumenta extraordinariamente. É o que acontece quando se dá liberdade no uso das tomadas de água.

Ossistemas SCADA (acrónimoinglêsque significa Supervisory Control And Data Acquisition) permitem a monitorização e telecomando de órgãos hidráulicos em tempo real. Por isso, têm vindo a ser usados, cada vez mais, mesmo a nível nacional, para melhorar a gestão dos canais com controlo por montante (Rijo,1999; Rijo, 2008 b; Rijo e Arranja, 2010; Rijo e Inácio, 2014; Rijo et al., 2005). O controlo de caudais nas admissões aos canais passa a ser realizado por telecomando em tempo real e a monitorização do estado hidráulico passa também a ser possível em tempo real, ganhando-se tempo, permitindo a gestão quantificada (na gestão tradicional, muitas vezes nem é possível estimar os caudais admitidos) e reduzindo as perdas de água associadas ao controlo tradicional de caudais.

A aplicação dos sistemas SCADA é a via mais económica e eficiente para a modernização de canais de rega, reduzindo as perdas de água associadas ao controlo tradicional de caudais e a mão de obra necessária à operação dos canais. O artigo faz uma descrição sumária das redes 
primária e secundária do Aproveitamento Hidroagrícola de Idanha-a-Nova (AHIN), ambas em canal e controladas por comportas AMIL, sendo os demais equipamentos também do tipo Neyrpic, nomeadamente as tomadas de água, na sua maioria do tipo módulo normalizado Neyrpic (SOREFAME, 1953). Em seguida, faz a apresentação do SCADA desenvolvido e implementado para a gestão e operação das duas redes do AHIN, incluindo alguns sinópticos, comunicações e as respetivas estações de campo. $\mathrm{O}$ artigo faz também a apresentação dos controladores digitais desenvolvidos para as admissões aos canais principais -controladores direto e de posição das comportas (para a operação de comportas) e controladores de caudal para a instalação de caudais prédefinidos e os correspondentes ajustamentos automáticos das comportas de admissão. A supervisão de níveis de água nas secções mais importantes e dos caudais nas descargas principais para o sistema de drenagem são também apresentadas.

\section{APROVEITAMENTO HIDROAGRÍCOLA DE IDANHA-A-NOVA}

O Aproveitamento Hidroagrícola de Idanhaa-Nova (AHIN) situa-se na campina da Idanha, no concelho Idanha-a-Nova, distrito de Castelo Branco, e beneficia uma área aproximada de $8200 h a$. É constituído pela Barragem Marechal Carmona e pelas infraestruturas da rede de rega e restantes estruturas que servem de apoio à exploração e conservação da obra de rega.

A exploração do AHIN está entregue à Associação de Regantes e Beneficiários de Idanha-a-Nova (ARBI).

O perímetro é composto por 2 grandes blocos de rega - bloco da Campina, com uma área aproximada de 5700ha, servido pelo Canal Condutor Geral (CCG), pelos distribuidores n. ${ }^{\circ 1}$ a 21 e pela estação elevatória (EE) do Ladoeiro; bloco do Aravil, com uma área aproximada de 2500ha, servido pelo Canal Condutor Geral do Aravil (CCGa), Canal Direito e Canal Esquerdo, este com os distribuidores $n^{\circ} 1$ a 5 e pela EE do Aravil, na admissão ao CCGa (Figura 2).

A rede primária de rega constitui o sistema adutor aos dois blocos de rega. É constituída por (Figura 2):
- CCG - Canal Condutor Geral - com início na Barragem Marechal Carmona e fim na EE do Ladoeiro; é dimensionado para um caudal de $7.38 \mathrm{~m}^{3} / \mathrm{s}$ no seu trecho inicial e tem um desenvolvimento total de $23701 \mathrm{~m}$.

- CCGcont - Canal Condutor Geral de continuação - é a continuação direta do CCG; desenvolve-se entre a EE do Ladoeiro e o distribuidor 19 (D19), junto à sede da ARBI; está dimensionado para um caudal de $1.58 \mathrm{~m}^{3} / \mathrm{s}$ e tem um comprimento de $2614 \mathrm{~m}$.

- CCGa - Canal Condutor Geral do Aravil - com início na EE do Aravil, termina no nó de derivação dos Canal Esquerdo (CE) e Canal Direito (CD); está dimensionado para o caudal de $2.275 \mathrm{~m}^{3} / \mathrm{s}$ e possui um comprimento de $807.5 \mathrm{~m}$.

A rede secundária de rega, com início na rede primária, para além da adução, tem já uma importante função de distribuição de água, sendo constituída por (Figura 2):

- No CCG - Distribuidores 1 a 17 (D1 a D17).

- No CCGcont - Distribuidores 18 a 21 (D18 a D21).

- No CCGa-CE, com os distribuidores 1, 2, 3, 4 e 5 do Aravil e o CD.

\section{SCADA DOS CANAIS PRIMÁRIOS E SECUNDÁRIOS DO AHIN}

\subsection{Apresentação geral}

OSCADA foi instalado e calibrado durante o ano de 2014. A fase última de calibração decorreu no final da campanha de rega desse ano.

Exigindo os canais do AHIN o controlo manual de caudais em todas as admissões, o primeiro objetivo do SCADA é o comando manual à distância dos órgãos de controlo de caudais nas admissões principais, canais primários e distribuidores principais (canais secundários), de modo a poder ajustar-se, em tempo real, os caudais admitidos aos caudais variáveis a distribuir.

O comando manual à distância dos órgãos de admissão aos canais é complementado por uma função de supervisão para definição 


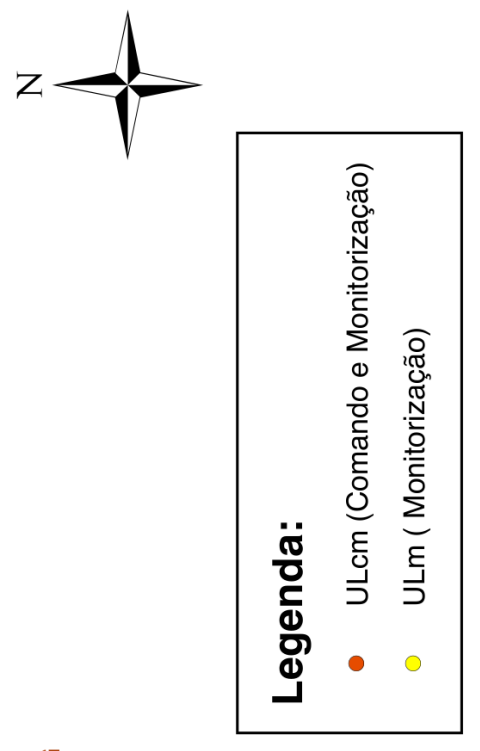

絗
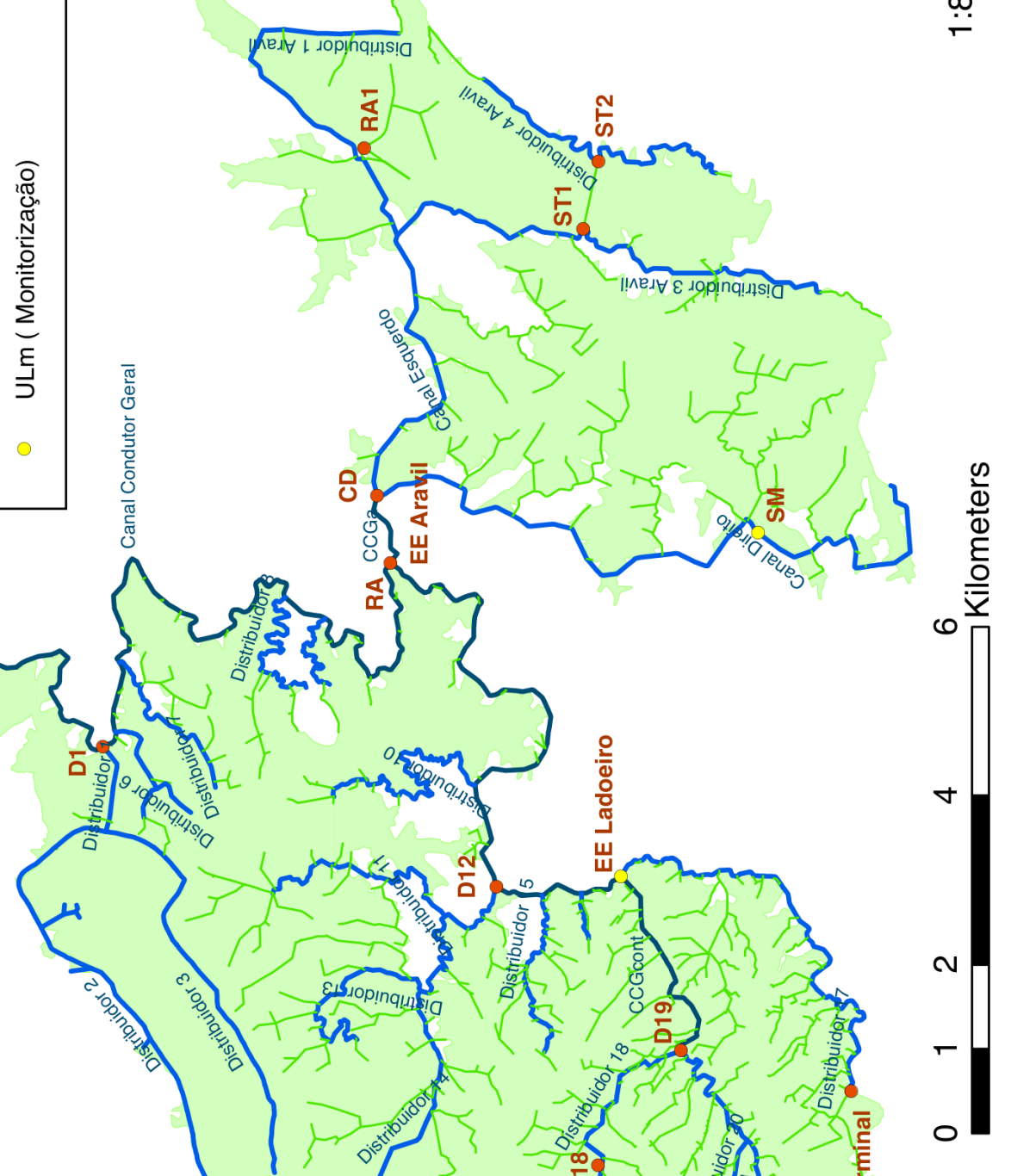

- 
de alarmes (riscos de galgamento de canais) também via SCADA - das alturas de água nas secções onde esse risco é maior e a montante e a jusante de comportas a telecomandar.

De modo a apoiar a tomada de decisão do responsável relativamente aos caudais a admitir, é importante poder dispor-se de informação, também em tempo real, dos caudais que saem para o sistema de drenagem, nomeadamente nas descargas principais. Esta função é ainda garantida pelo SCADA.

O SCADA é, basicamente, constituído pelos seguintes elementos:

- Centro de comando e supervisão - para o comando e a supervisão dos órgãos hidráulicos principais e a supervisão das alturas de água nas secções escolhidas;

- Unidades Locais (Estações de Campo) - para a recolha de informação local e o comando dos órgãos hidráulicos, obedecendo às ordens do centro de comando e supervisão ou trabalhando de modo independente;

- Sistema de comunicações - para a troca de dados e de informação entre o centro de comando e supervisão e as unidades locais.

O centro de comando e supervisão está instalado na sede da ARBI. Tem como funções principais:

- O acesso remoto ao estado de cada uma das unidades locais e as respetivas supervisões, usando sinópticos atualizados "on-line" a partir da base de dados, de modo a que o operador possa aceder ao estado e comandar qualquer equipamento das unidades locais;

- O comando remoto dos equipados selecionados;

- A gestão de alarmes, com impressão em papel contínuo e envio de mensagens escritas (SMS) para o telemóvel do operador caso este não se encontre presente no centro de controlo; o tipo de alarmes a enviar foi configurado de acordo com o seu nível de urgência;

- A análise e arquivamento de dados;

- A gestão do sistema de adução em tempo real.
Em caso de falha de comunicações, é dado um alarme, sendo identificada(s) a(s) respectiva(s) estação(ões) de campo em falha.

O operador tem acesso aos dados, regra geral via sinópticos, que permitem uma leitura rápida do estado das estações de campo.

As diferentes estações de campo estão equipadas com autómatos de modo a terem capacidade de decisão local e receberem ordens do sistema de supervisão. Assim, mesmo que haja falha de comunicação com o centro de comando e supervisão, os autómatos locais permitem a implementação de decisões a nível local.

Os princípios de base da gestão hidráulica dos canais não se alteraram com a implementação do SCADA. A gestão operacional dos canais continuaasermuitopersonalizada, continuando a ser muito importante a experiência de campo acumulada, como é característico dos canais com controlo por montante. O SCADA é uma ferramenta poderosa na modernização da gestão tradicional, que se irá refletir na economia de água, de mão-de-obra e de energia, mas não mudará a lógica de gestão, daí que a definição dos pontos ou secções a equipar com estações de campo tivesse sido realizada em colaboração com os responsáveis da gestão hidráulica da rede.

O SCADA tem a configuração geral que se apresenta na Figura 3.

\subsection{Unidades locais}

As unidades locais recebem informações (sinais elétricos) dos sensores (alturas de água, posição das comportas, caudais) convertendoas para variáveis com significado físico e armazenando-as numa base de dados própria (data logging). A conversão pode resultar da aplicação de uma escala linear (altura de água, por exemplo) ou resultar de um algoritmo de cálculo (caudal, por exemplo).

Periodicamente, esses dados são transferidos para a central de comando e supervisão, de modo a estarem sempre disponíveis para consulta e análise. A sincronização ocorre também sempre que se verifique uma condição de alarme.

As unidades locais são, essencialmente, unidades de comando e de telemetria 


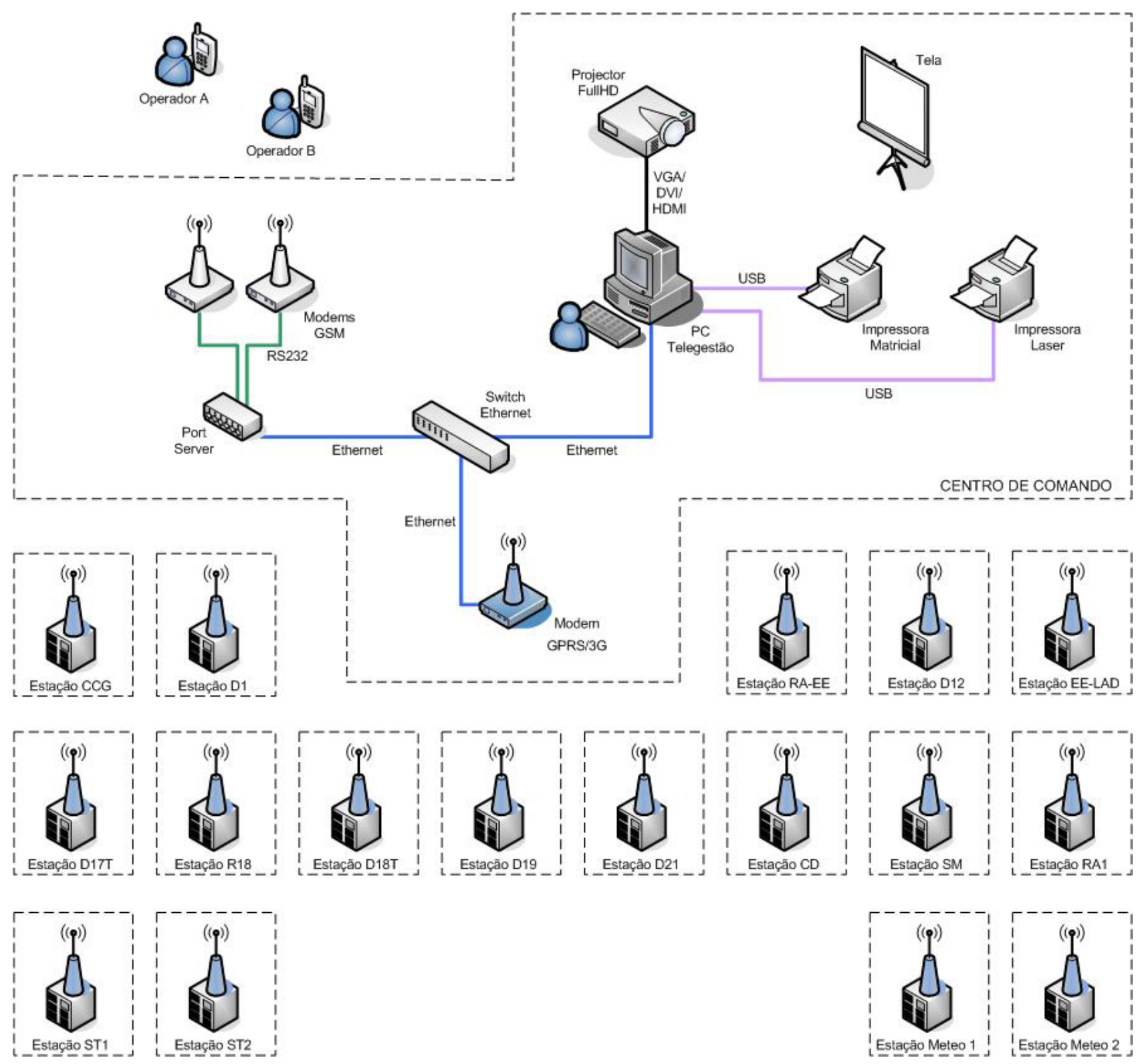

Figura 3. Configuração geral do SCADA do AHIN.

ou unidades de telemetria (supervisão/ monitorização), sendo constituídas genericamente por:

- autómato programável - para gestão da unidade local, processamento de informação e troca de dados com o centro de comando e supervisão;

- modem de comunicações - para permitir a comunicação entre o autómato local e o centro de comando e supervisão;

- interface homem-máquina (HMI) - para permitir o acesso a informação local, comando e parametrização local do funcionamento do autómato;

- comportas com atuadores elétricos - para controlarem o caudal admitido/distribuído;

- equipamento de proteção e comando - para acionamento dos atuadores;

- sensores de posição das comportas e de altura de água - para fornecerem a indicação do estado das comportas e do canal;

- quadro de força motriz - para alimentar o autómato, atuadores e restantes equipamentos; 
- baterias - para armazenamento de energia, de modo a garantir a continuidade do funcionamento da unidade local mesmo com falhas na alimentação de energia;

- abrigo - para albergar os equipamentos.

A Tabela 1 caracteriza sumariamente os locais escolhidos para integrarem estações de campo do SCADA.

A Figura 2 e a Tabela 2 apresentam a localização e tipologia das 16 estações de campo projetadas e instaladas.

As unidades de campo do SCADA são de dois tipos:

- ULcm-Unidadelocalcomcontrolo(comando ou atuação) e monitorização (supervisão ou telemetria); possui equipamento para telecomando, função de data logging e posterior envio dos dados para o centro de comando e supervisão e podem reunir as funções que seguem:

monitorização de alturas de água - a montante $\left(h_{1}\right)$ ou a jusante $\left(h_{2}\right)$ de um órgão ou estrutura hidráulica (limpa grelhas, comporta, descarregador frontal ou lateral);

monitorização de abertura de comportas (a);

> monitorização de caudais para o sistema de drenagem através de descarregador $\left(Q_{1}\right)$ e/ou com caudalímetro $\left(Q_{2}\right)$;

$>$ controlo de caudais $(Q)$ - de admissão a distribuidor, reservatório ou de saída através de comporta.

- ULm - Unidade local de monitorização (supervisão ou telemetria); não possui equipamento para telecomando, tendo apenas a função de data logging e posterior envio dos dados para o centro de comando e supervisão e reúne uma ou mais das funções que seguem:

> monitorização de alturas de água - a montante $\left(h_{1}\right)$ ou a jusante $\left(h_{2}\right)$ de uma máquina, órgão ou estrutura hidráulica (limpa grelhas, comporta, descarregador frontal ou lateral);

$>$ monitorização de abertura de comportas $(a)$; monitorização de caudais para o sistema de drenagem através de descarregador $\left(Q_{1}\right)$ e/ou com caudalímetro $\left(Q_{2}\right)$.

\subsection{Controladores digitais instalados nos autómatos}

\section{Controlador Direto para uma ou mais comportas (D,} Tabela 2)

O controlo direto de uma comporta consiste na capacidade de um utilizador - através de uma $H M I$ - acionar um botão para a abrir, fechar ou parar numa determinada posição. Estas ações de comando podem ser arbitrárias (decisão do operador humano) ou resultar de um controlador automático.

A sua aplicabilidade prática está relacionada com atividades de manutenção/testes no canal ou nos equipamentos, necessidade de atuar de imediato no canal e também para serem usados como parte final de controladores mais sofisticados instalados na cadeia de controlo, como são os casos dos controladores de posição e de caudal.

A Figura 4 apresenta o controlador de caudal para as situações em que há duas comportas. O controlador direto recebe do controlador de caudal as aberturas a instalar em cada comporta, $a_{1 r}$ e $a_{2 r}$. O controlador direto, em função do estado de cada comporta $\left(a_{1 \text { status }} \mathrm{e}\right.$ $\left.a_{2 \text { status }}\right)$, envia as ordens de comando para os atuadores de cada comporta, respetivamente, $a_{1}$ e $a_{2}$. É garantido um tempo de paragem mínimo para cada motor $\left(\Delta T_{1 O F F}\right.$ e $\left(\Delta T_{2 O F F}\right)$, calculado com base no número de arranques por hora permitidos pelos motores.

\section{Controlador de Posição para uma ou mais comportas ( $\mathrm{P}$, Tabela 2)}

No controlador de posição, a ordem de comando da(s) comporta(s) pode ser enviada por uma $\mathrm{HMI}$, pelo PC Central (SCADA) ou através do PLC local. Para o caso de duas comportas, o controlador de posição compara a nova posição requerida para cada comporta com a posição atual ( $a_{1 \text { med }}$ e $a_{2 m e d}$, Figura 4). Dessa comparação, resulta uma ordem de comando (Abrir/Fechar/Stop), $a_{1 r}$ e $a_{2 r}$ a transmitir ao controlador direto. 
Tabela 1. Caracterização preliminar dos locais a integrar em estações de campo do SCADA do AHIN

\begin{tabular}{|c|c|c|c|c|c|c|c|c|}
\hline \multirow[t]{2}{*}{ Locais } & \multicolumn{2}{|c|}{ Caudal } & \multicolumn{5}{|c|}{ Admissão } & \multirow[t]{2}{*}{ OBS. } \\
\hline & $\begin{array}{l}\text { Admissão } \\
\qquad\left(m^{3} s^{-1}\right)\end{array}$ & $\begin{array}{l}\text { Canal } \\
\left(m^{3} s^{-1}\right)\end{array}$ & $\begin{array}{l}\text { Largura útil } \\
\qquad(m)\end{array}$ & $\begin{array}{l}\text { Largura total } \\
\qquad(m)\end{array}$ & Módulo & $\begin{array}{l}\text { Altura do } \\
\text { módulo(*) } \\
\qquad(m)\end{array}$ & $\begin{array}{l}\text { Altura do } \\
\text { canal }^{(* *)}(m)\end{array}$ & \\
\hline CCG & 7.38 & 7.38 & 5.50 & -- & 2 comportas & --- & 1.23 & $\begin{array}{l}\text { Esc. livre/subm.; com } \\
\text { energia }\end{array}$ \\
\hline D1 & -- & 0.784 & 1.40 & 1.75 & 5 comportas & --- & 1.07 & $\begin{array}{l}\text { Esc. livre/subm.; sem } \\
\text { energia }\end{array}$ \\
\hline RA-EE Aravil & -- & 2.275 & 1.50 & 1.68 & 1 comporta & --- & 2.30 & $\begin{array}{l}\text { Esc. livre/subm., } \\
\text { inversão de caudal; } \\
\text { com energia }\end{array}$ \\
\hline D12 & --- & 1.134 & 1.95 & 2.12 & $\begin{array}{l}3 \text { orifícios com } \\
\text { comportas }\end{array}$ & --- & 1.45 & $\begin{array}{l}\text { Esc. livre/subm.; sem } \\
\text { energia }\end{array}$ \\
\hline EE Ladoeiro & --- & --- & --- & --- & --- & --- & --- & Com energia \\
\hline D17 Terminal & 0.065 & 0.065 & 0.065 & 0.067 & $X_{1}-M 3-65$ & 0.58 & 0.42 & Esc. livre; sem energia \\
\hline R18-adução & 2.30 & 2.30 & $2 \times 0.80$ & 2.0 & 2 comportas & --- & 1.80 & $\begin{array}{l}\text { Esc. livre/subm.; sem } \\
\text { energia }\end{array}$ \\
\hline R18-tomada & $1.10-0.66$ & -- & --- & -- & comporta $\varnothing 800 \mathrm{~mm}$ & --- & --- & $\begin{array}{l}\text { Esc. em pressão; sem } \\
\text { energia }\end{array}$ \\
\hline D18 Terminal & 1.025 & 1.025 & 0.94 & 1.05 & 2 comportas & -- & 1.05 & $\begin{array}{l}\text { Esc. livre/submerso; } \\
\text { sem energia }\end{array}$ \\
\hline D19 & 0.288 & 0.288 & 1.00 & 1.87 & $\begin{array}{l}1 \text { orifício com } 2 \\
\text { comportas }\end{array}$ & --- & 1.50 & $\begin{array}{l}\text { Esc. livre/subm.; com } \\
\text { energia }\end{array}$ \\
\hline D21 & -- & 0.111 & --- & --- & --- & --- & 0.75 & Sem energia \\
\hline R21-tomada & $0.19-0.16$ & --- & --- & --- & comporta Ø400 mm & --- & --- & $\begin{array}{l}\text { Esc. em pressão; sem } \\
\text { energia }\end{array}$ \\
\hline$C D$ & 0.608 & 0.608 & --- & -- & Estrutura & --- & 1.50 & $\begin{array}{l}\text { Esc. livre/submerso; } \\
\text { sem energia }\end{array}$ \\
\hline SM & -- & 0.608 & -- & -- & -- & -- & 1.40 & Sem energia \\
\hline RA1-adução & 1.265 & 1.265 & 1.00 & 1.0 & 1 comporta & --- & 2.50 & $\begin{array}{l}\text { Esc. livre/submerso; } \\
\text { sem energia }\end{array}$ \\
\hline RA1-jusante & 1.265 & 1.265 & $2 \times 1.00$ & 2.90 & 2 comportas & --- & 1.35 & $\begin{array}{l}\text { Esc. livre/submerso; } \\
\text { sem energia }\end{array}$ \\
\hline RA1-tomada & 0.30 & --- & --- & --- & comporta $\varnothing 700 \mathrm{~mm}$ & --- & --- & $\begin{array}{l}\text { Esc. em pressão; sem } \\
\text { energia }\end{array}$ \\
\hline $\begin{array}{l}\text { ST1 } \\
\text { ST2-D4 }\end{array}$ & $\begin{array}{l}0.493 \\
0.203\end{array}$ & $\begin{array}{l}0.493 \\
0.203\end{array}$ & $\begin{array}{l}1.04 \\
1.32\end{array}$ & $\begin{array}{l}1.34 \\
1.94\end{array}$ & $\begin{array}{l}1 \text { comporta } \\
2 \text { comportas }\end{array}$ & --- & 0.75 & $\begin{array}{l}\text { Esc. livre; sem energia } \\
\text { Esc. livre/subm.; sem } \\
\text { energia }\end{array}$ \\
\hline ST2-D5 & 0.121 & 0.121 & 0.80 & 1.40 & 2 comportas & --- & 0.67 & $\begin{array}{l}\text { Esc. livre/subm.; sem } \\
\text { energia }\end{array}$ \\
\hline
\end{tabular}

(*) Desde a crista da soleira até ao topo do modulo Neyrpic.

(** ) Desde o rasto do canal ou da crista da soleira Neyrpic até à banqueta do canal. 
Tabela 2. Caracterização sumária das estações de campo do SCADA do AHIN

\begin{tabular}{|c|c|c|c|c|c|c|c|c|c|}
\hline Estação & $\begin{array}{l}\text { Localização da } \\
\text { estação }\end{array}$ & $\begin{array}{c}\text { Tipo } \\
\text { estação }\end{array}$ & $\begin{array}{c}\mathrm{N}^{\circ} \\
\text { Comportas }\end{array}$ & $\begin{array}{l}\text { Ressalto } \\
\text { Hidráulico }\end{array}$ & Monitorização & Descarreg. & $\begin{array}{l}\text { Sensores } \\
\text { Caudal }\end{array}$ & Controladores & Energia \\
\hline \multicolumn{10}{|c|}{ Canal Condutor Geral } \\
\hline CCG & Admis. CCG & ULm & --- & --- & $(3 *)$ & --- & --- & --- & Rede \\
\hline D1 & $\begin{array}{l}\text { Admis. } \\
\text { Distribuidor } 1\end{array}$ & ULcm & 1 & Livre/subm. & $h_{1^{\prime}} h_{2^{\prime}} a$ & --- & --- & $D, P, Q$ & $\begin{array}{l}\text { Painel } \\
\text { solar }\end{array}$ \\
\hline RA-EE Aravil & EE Aravil & ULcm & $1\left(^{*}\right)$ & Livre/subm. & $h_{1}, h_{2^{\prime}} a, Q_{1}, Q_{2}$ & 2 & 1 & $D, P, Q\left({ }^{5 *}\right)$ & Rede \\
\hline D12 & $\begin{array}{l}\text { Admis. } \\
\text { Distribuidor } 12\end{array}$ & ULcm & 2 & Livre/subm. & $h_{1}, h_{2^{\prime}} a$ & -- & -- & $D, P, Q$ & Rede \\
\hline EE Ladoeiro & EE Ladoeiro & ULm & -- & -- & $h_{1}, h_{2^{\prime}} Q_{1}, Q_{2}$ & 2 & 1 & -- & Rede \\
\hline $\begin{array}{l}\text { D17 } \\
\text { Terminal }\end{array}$ & $\begin{array}{l}\text { Admis. } \\
\text { Distribuidor } 17\end{array}$ & ULcm & 1 & Livre & $h_{1}, a, Q_{1}$ & 2 & --- & $D, P, Q$ & $\begin{array}{l}\text { Painel } \\
\text { solar }\end{array}$ \\
\hline \multicolumn{10}{|c|}{ Canal Condutor Geral continuação } \\
\hline $\mathrm{R} 18$ & $\begin{array}{l}\text { Reservatório } \\
18\end{array}$ & ULcm & $\begin{array}{c}2\left({ }^{*}\right)+1\left(^{*}\right) \\
\left({ }^{* *}\right)\end{array}$ & Livre/subm. & $h_{1^{\prime}} h_{2^{\prime}} a, Q_{1^{\prime}} Q_{2}$ & 1 & $1+1\left({ }^{4 *}\right)$ & $D, P, Q$ & Rede \\
\hline $\begin{array}{l}\text { D18 } \\
\text { Terminal }\end{array}$ & $\begin{array}{l}\text { Termin. } \\
\text { Distribuidor } 18\end{array}$ & ULcm & 1 & Livre/subm. & $h_{1}, h_{2^{\prime}} a$ & 1 & --- & $D, P, Q$ & $\begin{array}{l}\text { Painel } \\
\text { solar }\end{array}$ \\
\hline D19 & $\begin{array}{l}\text { Admis. } \\
\text { Distribuidor } 19\end{array}$ & ULcm & 1 & Livre/subm. & $h_{1}, h_{2}, a, Q_{2}$ & -- & 1 & $D, P, Q$ & Rede \\
\hline D21 & Distribuidor 21 & ULm & -- & -- & $h_{1}, Q_{2}$ & -- & 1 & --- & $\begin{array}{l}\text { Painel } \\
\text { solar }\end{array}$ \\
\hline $\mathrm{R} 21$ & $\begin{array}{l}\text { Reservatório } \\
21\end{array}$ & ULcm & $1\left({ }^{*}\right)(* *)$ & -- & $h_{1}, w, Q_{1}$ & 1 & --- & $D, P, Q$ & Rede \\
\hline \multicolumn{10}{|c|}{ Canal Condutor Geral do Aravil } \\
\hline$C D$ & $\begin{array}{l}\text { Admis. Canal } \\
\text { Direito }\end{array}$ & ULcm & 2 & Livre/subm. & $h_{1}, h_{2^{\prime}} a$ & --- & --- & $D, P, Q$ & Rede \\
\hline SM & $\begin{array}{l}\text { Sifão } \\
\text { Mascarenha }\end{array}$ & ULm & -- & -- & $h_{1}, Q_{1}, Q_{2}$ & 1 & 1 & --- & $\begin{array}{l}\text { Painel } \\
\text { solar }\end{array}$ \\
\hline RA1 & $\begin{array}{l}\text { Reservatório } 1 \\
\text { do Aravil }\end{array}$ & ULcm & $\begin{array}{c}3(*)+1\left(^{*}\right) \\
\left({ }^{* *}\right)\end{array}$ & Livre/subm. & $h_{1}, h_{2^{\prime}} a, Q_{2}$ & 1 & --- & $D, P, Q$ & Rede \\
\hline ST1 & $\begin{array}{l}\text { Admissão } \\
\text { Sifão Toula }\end{array}$ & ULcm & 1 & Livre & $h_{1}, a, Q_{1}$ & 1 & -- & $D, P, Q$ & $\begin{array}{l}\text { Painel } \\
\text { solar }\end{array}$ \\
\hline $\mathrm{ST} 2$ & $\begin{array}{l}\text { Saída Sifão } \\
\text { Toula }\end{array}$ & ULcm & $1+1$ & Livre/subm. & $h_{1}, h_{2}, a$ & -- & --- & $D, P, Q$ & $\begin{array}{l}\text { Painel } \\
\text { solar }\end{array}$ \\
\hline
\end{tabular}

(*) Já instalada

(**) Comporta mural da tomada de água; escoamento em pressão

(3*) Leitura de sinais de SCADA já existente.

$\left(4^{*}\right)$ Medidor de caudal eletromagnético na conduta da tomada de água

$\left(5^{*}\right)$ Com possibilidade de inversão do caudal 
As saídas do controlador de posição são as entradas do controlador direto. Sendo este o controlador mais simples, é, porém, o mais importante.

\section{Controlador de Caudal para uma ou mais comportas ( $Q$, Tabela 2)}

A Figura 4 apresenta o controlador de caudal para duas comportas, conforme já assinalado. O controlador recebe a referência ou ordem de caudal $Q_{r}$, caudal total para as duas comportas. Tendo em conta as alturas de águas a montante e a jusante das comportas, $h_{1}$ e $h_{2}$ e as aberturas das mesmas ( $a_{1 m e d}$ e $a_{2 m e d}$ ), o controlador calcula o caudal $Q$, caudal atual ou que está a passar naquela situação nas duas comportas.

O cálculo do desvio $\left(e_{O}\right)$ entre o caudal atual $Q$, dado pela equação de vazão da comporta, e o novo caudal a instalar $Q_{r}$ pode originar três situações distintas:

- Se $e_{Q}>\Delta Q$ (erro positivo: necessidade de aumentar o caudal) - Abre comportas (as duas em simultâneo na situação normal, ou, havendo avaria numa delas, a outra deverá compensar a diferença de abertura desta);

- Se $e_{Q}<\Delta Q$ (erro negativo: necessidade de diminuir o caudal) - Fechar comportas;

- Se $e_{Q}=0$ (erro nulo: necessidade de manter o caudal) - Imobiliza comportas.
Tal como acontece no controlador de posição, a saída do controlador de caudal - $a_{1 r}$ e $a_{2 r}-$ representa as ordens de abrir/fechar comportas transmitidas ao controlador direto.

Cálculo do caudal $\boldsymbol{Q}$ - As estruturas de controlo de caudais nas admissões aos canais podem funcionar como descarregadores (comportas acima da superfície livre) ou como comportas associadas ou não a soleiras e, para cada situação, haver ainda situações de escoamento livre (ressalto hidráulico livre a jusante), escoamento parcialmente submerso e escoamento totalmente submerso.

O algoritmo geral de cálculo de $Q$ usado nos autómatos garante a continuidade das soluções para todo o domínio de funcionamento das estruturas, isto é, sem descontinuidades numéricas. Esse algoritmo é apresentado em Rijo e Miranda (2016).

\subsection{Comunicações}

A arquitetura de comunicações entre o centro de comando e as unidades locais é baseada numa APN de um operador móvel (TMN) sobre GPRS/UMTS. Através desta solução, o sistema fica dotado de uma rede IP, com as vantagens correspondentes.

As comunicações são suportadas pelo protocolo IEC60870-5-104. Este protocolo, baseado no

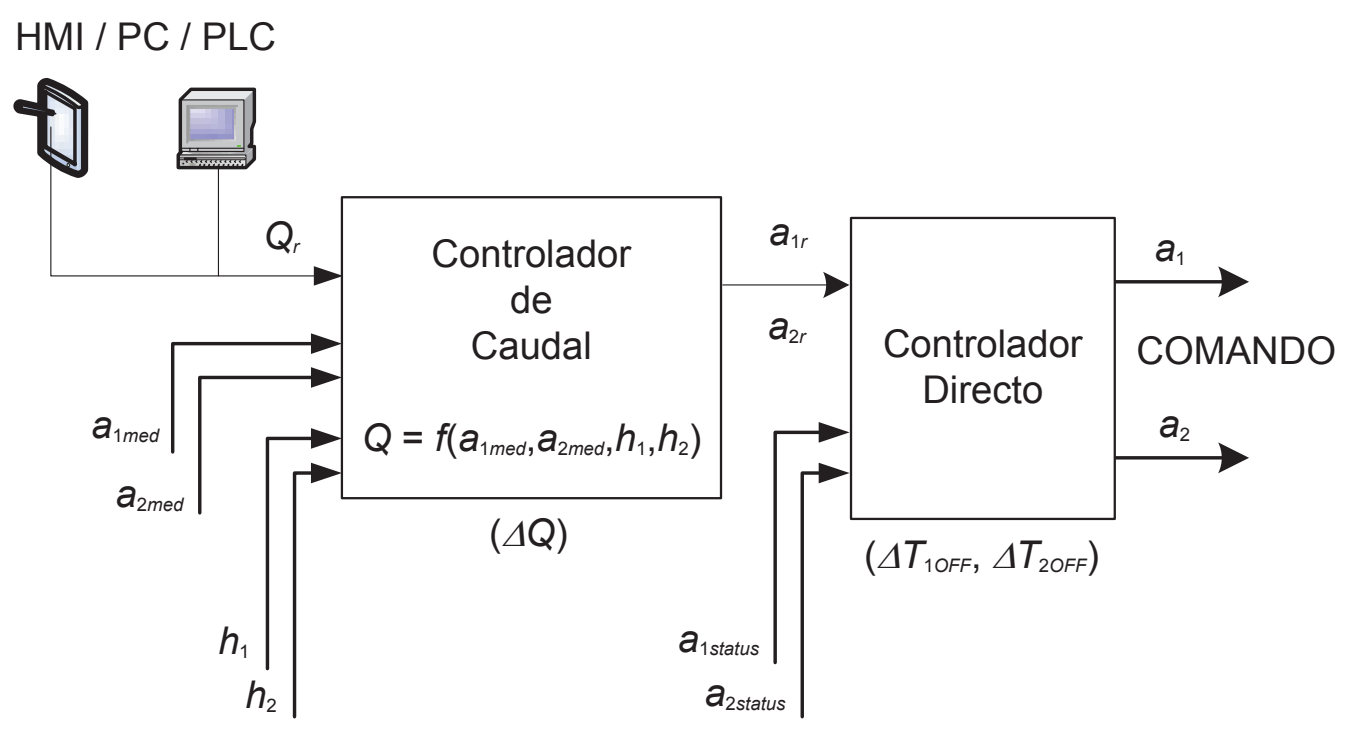

Figura 4. Controlador de caudal para duas comportas (Rijo, 2010). 
modelo OSI "Open System Interconnection", foi criado para fazer face às exigências inerentes a instalações de distribuição de energia, tendo vindo a ser implementado gradualmente e com sucesso em instalações de distribuição de água.

Neste tipo de aplicações, a grande vantagem do protocolo é que não é necessária uma leitura permanente de dados por parte do SCADA. A transmissão de dados para o SCADA acontece somente mediante a ocorrência de eventos ou alarmes nas estações, minimizando muito o volume de dados na rede. Apesar disto, se necessário, é possível efetuar interrogações sobre as informações e dados das instalações partir do sistema de supervisão.

\subsection{Exemplos de sinópticos}

A Figura 5 apresenta o sinóptico principal da aplicação SCADA. Apresenta uma vista geral da rede de rega, com indicação dos vários canais existentes. Clicando sobre um canal, pode aceder-se à página de cada uma das estações de campo desse canal.

A janela principal encontra-se dividida em quatro partes:
- Barra de título (superior) - contém o título da página e a data e hora atual

- Barra de navegação (esquerda) - permite navegar entre as várias páginas do SCADA, sendo acessíveis as seguintes janelas:

Rede primária - Janela principal

- Canais (para aceso direto a cada uma das estações de campo do SCADA)

- CCG - Canal Condutor Geral

- Campina - Bloco da Campina

- Aravil - Bloco do Aravil

Meteo - Estações Meteorológicas

Alarmes

$>$ Eventos

> Gráficos

$>$ Relatórios

Ferramentas

Comunicações

Sistema

- Barra de informação (inferior) - tem a indicação permanente de quais os alarmes ocorridos que ainda não foram reconhecidos.

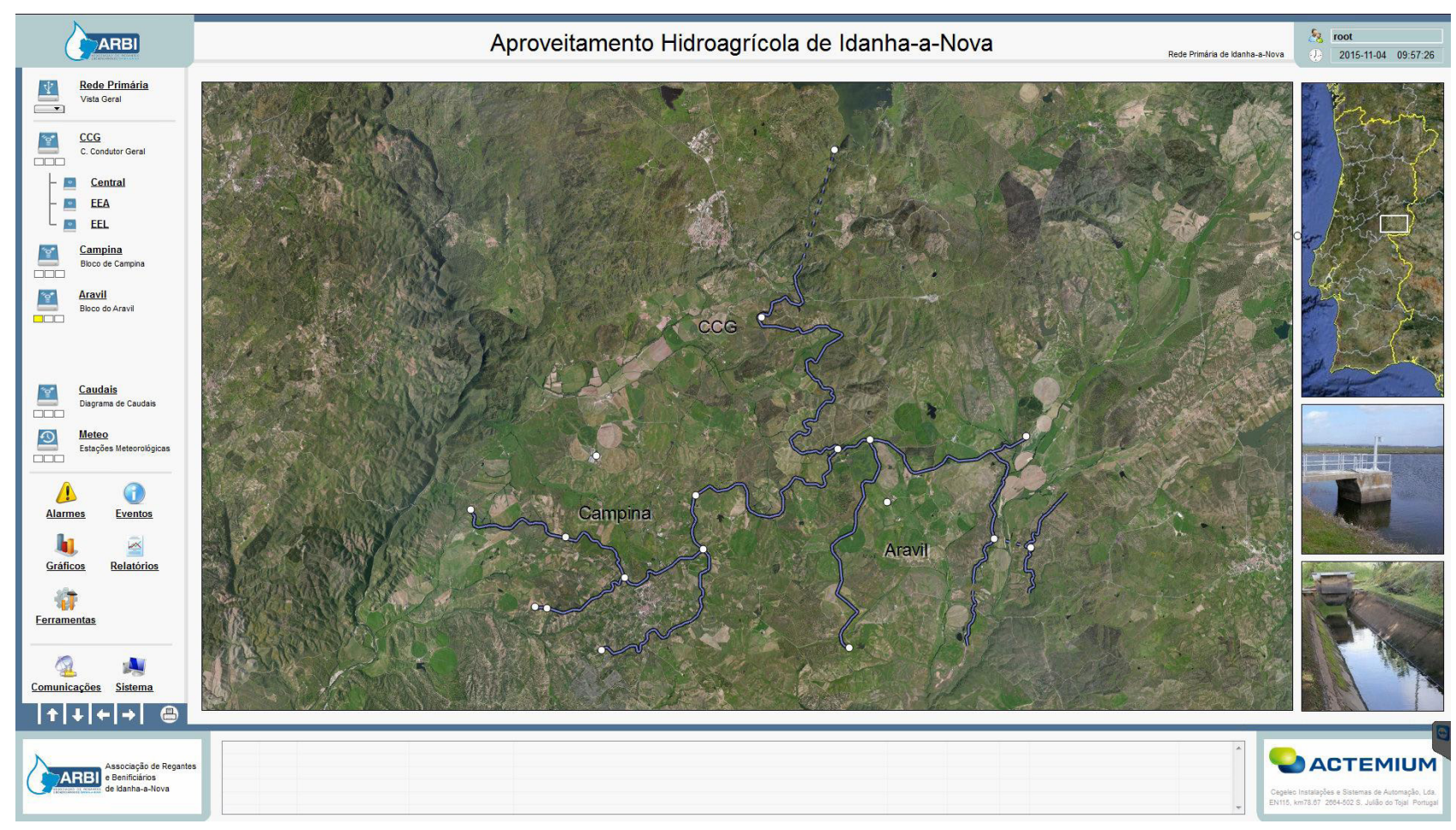

Figura 5. Janela principal do SCADA do AHIN. 
- Área principal - Ao selecionar uma janela, esta é mostrada na área principal da aplicação. É o caso do sinóptico da estação D12, que se apresenta na Figura 6, que apresenta, nomeadamente, as alturas de água a montante e jusante das duas comportas de admissão ao distribuidor, o caudal e a percentagem de abertura de cada comporta. Na Figura 6, são ainda visíveis os controladores que se podem definir e os horários de rega (calendário de caudais a colocar nas comportas).

\section{CONSIDERAÇÕES FINAIS}

OssistemasSCADAexistemháalgumas décadas. Contudo, a grande maioria das aplicações a canais de rega foram concretizadas nos últimos 20 anos. Só na parte oeste dos EUA, instalaramse, na penúltima década, cerca de 150 sistemas SCADA em canais de rega (Burt, 2005). O desenvolvimento recente destas aplicações tem dado origem a realizações frequentes de conferências científicas internacionais sobre o tema, na sua maioria organizadas pela "US Committee on Irrigation and Drainage", que têm juntado investigadores, gestores de perímetros de rega e especialistas de sistemas
SCADA. No País, fez-se a primeira aplicação ao Canal Condutor Geral do Aproveitamento Hidroagrícola do Vale do Sorraia, há cerca de 25 anos.

Estas aplicações são, quase sempre, a primeira opção na modernização dos canais de rega com controlo por montante, pelas vantagens comparativas com outras soluções e atendendo ao facto de os investimentos envolvidos serem baixos e decrescentes, nomeadamente os relativos aos equipamentos eletrónicos e software. A título de exemplo, pode afirmarse que a aplicação apresentada teve um custo total abaixo de 1,2 milhões de euros.

Os sistemas SCADA são, só por si, ferramentas de gestão muito importantes, permitindo melhorar a qualidade de serviço, economizar água, poupar mão-de-obra na operação dos canais e ganhar tempo e energia.

A calibração da aplicação SCADA apresentada faz-se em Rijo e Miranda (2016).

\section{AGRADECIMENTOS}

Os autores agradecem à ARBI a disponibilização dos elementos de projeto para o presente artigo.

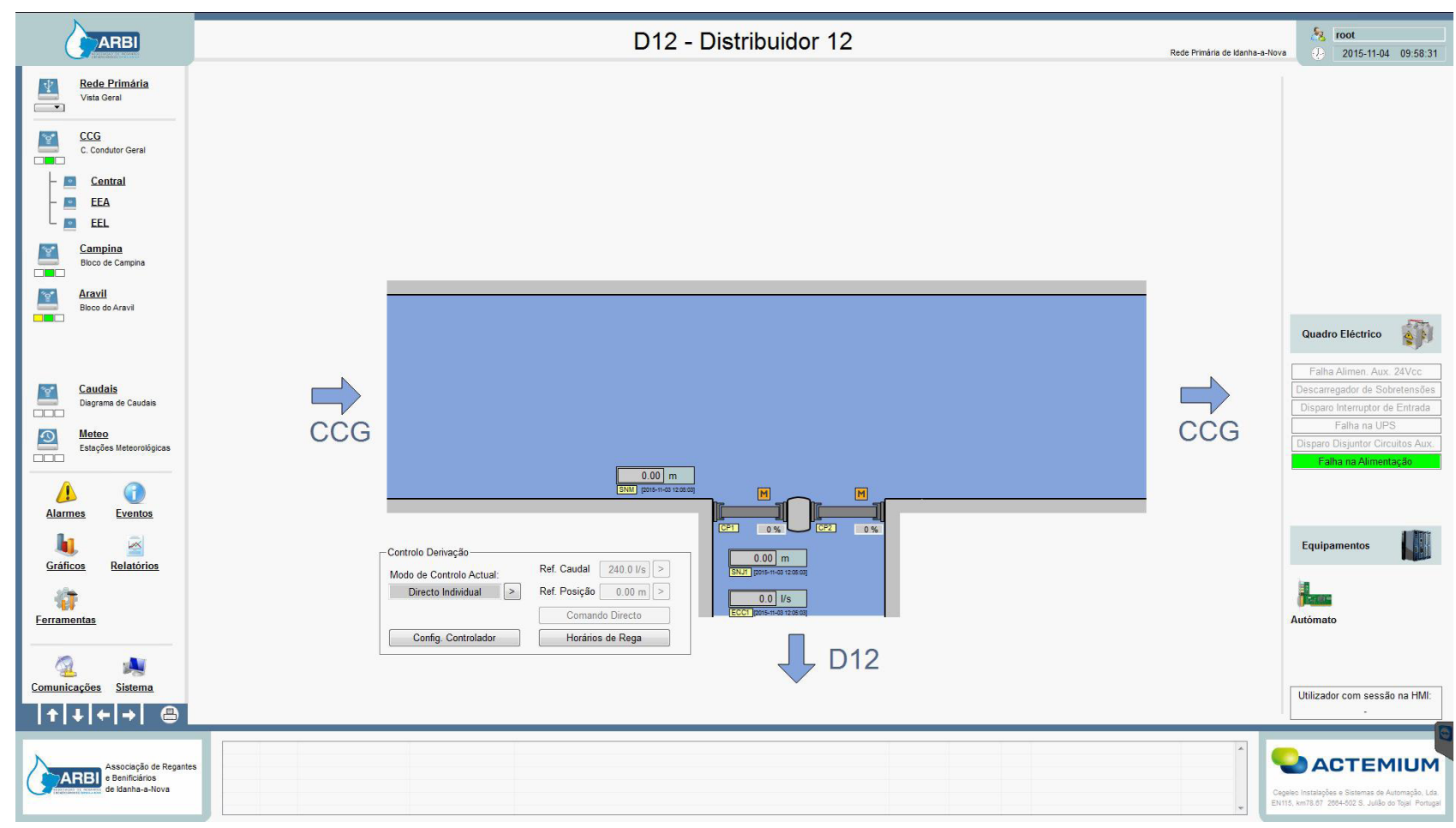

Figura 6. Janela de estação de campo D12 


\section{BIBLIOGRAFIA}

ALSTHOM - AMIL gate. Alsthom fluides, Note $n^{\circ}$ A6501A, Services Techniques et Commerciaux, Alsthom-Fluides, La Courneuve, France.

Burt, C. (2005) - Overview of Supervisory Control and Data Acquisition (SCADA). In: C. M. Burt \& S.S. Anderson (eds), SCADA and Related Technologies for Irrigation District Modernization, pp. 1-9, USCID Publisher, Denver, USA, ISBN: 1-887903-19-4.

EDIA (s/d) - Sistema Global de Rega. Empresa de Desenvolvimento e Infra-Estruturas do Alqueva, S.A., Beja, Portugal. In: http://www. edia.pt/pt/o-que-e-o-alqueva/sistema-globalde-rega/106 (acedido em Outubro de 2015).

Rijo, M. (2010) - Canais de Adução. Projecto, Operação, Controlo e Modernização. 531p., Edições Sílabo, Lisboa, Portugal. ISBN: 978-972618-615-1.

Rijo, M. (2008 a) - Modernização e controlo automático de sistemas de rega em canal. Recursos Hídricos, Vol. 29 (1), 87-100.

Rijo, M. (2008 b) - Design and field tuning of an upstream controlled canal network SCADA. Irrigation and Drainge, Vol. 57, 123-137.

Rijo, M. (1999) - SCADA of an Upstream Controlled Irrigation Canal System. In: A.J. Clemmens \& S.S. Anderson (eds.), Modernization of Irrigation Water Delivery Systems, pp. 123-136, USCID Publisher, Denver, USA, ISBN: 1-887903-07-0.
Rijo, M. \& Arranja, C. (2010) - Supervision and Automatic Control of an Irrigation Canal. Journal of Irrigation \& Drainage Engineering, American Society of Civil Engineers, Vol. 136 (1), 3-10.

Rijo, M. \& Inácio, B. (2014) - Modernização de canais de rega. Uma aplicação. CLME'2014 Congresso Luso-Moçambicano de Engenharia, CAP XVI - Hidráulica, Hidrologia e Recursos Hídricos num contexto de mudança: do risco à gestão sustentável, Inhambane, Moçambique, Gomes, J.F. et al. (eds.), pp. 321-322.

Rijo, M., Lanhoso A. \& Nunes, M. (2005) - Design and Implementation of an Irrigation Canal SCADA. In: C. M. Burt \& S.S. Anderson (eds), SCADA and Related Technologies for Irrigation District Modernization, pp. 23-32, USCID Publisher, Denver, USA, ISBN: 1-887903-19-4.

Rijo, M. \& Miranda, C. (2016) - Supervisão e controlo de um sistema de canais de rega. Parte II - Calibração hidráulica. Recursos Hídricos (Lisboa) (enviado para publicação).

SIR (s/d) - Área infra-estruturada - Total Nacional 2012. Sistema de Informação do Regadio, Autoridade Nacional do Regadio, Direção Geral de Agricultura e Dsenvolvimento Rural, Lisboa, Portugal. In: http://sir.dgadr.pt/ exp-infra (acedido em Outubro de 2015).

SOREFAME (1953) - Material de rega - Neyrpic. Catálogo RL86, Sorefame, Lisboa 